\title{
Auriculotherapy and reducing chronic musculoskeletal pain: integrative review
}

\author{
Auriculoterapia e redução da dor musculoesquelética crônica: revisão integrativa \\ Auriculoterapia y reducción del dolor musculoesquelético crónico: revisión integrativa
}

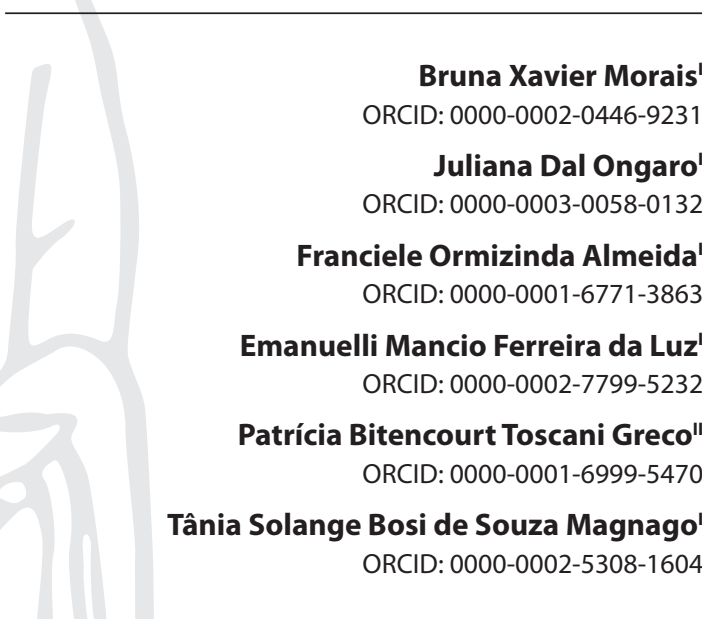

'Universidade Federal de Santa Maria. Santa Maria, Rio Grande do Sul, Brazil. "Universidade Regional Integrada do Alto Uruguai e das Missões. Santiago, Rio Grande do Sul, Brazil.

How to cite this article: Morais BX, Ongaro JD, Almeida FO, Luz EMF, Greco PBT Magnago TSBS. Auriculotherapy and reducing chronic musculoskeletal pain: integrative review.

Rev Bras Enferm. 2020;73(Suppl 6):e20190394. doi: http://dx.doi.org/10.1590/0034-7167-2019-0394

\section{Corresponding author:}

Bruna Xavier Morais

E-mail: bruna_morais100@hotmail.com

EDITOR IN CHIEF: Dulce Barbosa ASSOCIATE EDITOR: Fátima Helena Espírito Santo

\section{ABSTRACT}

Objectives: to describe the scientific evidence on the use of auriculotherapy to reduce chronic musculoskeletal pain in adults and the elderly. Methods: integrative literature review conducted in the Latin American and Caribbean Health Sciences, SciVerse Scopus and MEDLINE databases (via National Library of Medicine), in March 2019, with no time frame. Results: 14 original scientific articles were analyzed, $64.3 \%$ of which were classified with level of evidence 2. All productions show the benefit of auriculotherapy in reducing chronic musculoskeletal pain, especially in the lumbar spine (42.9\%). The treatment was developed in one or more sessions, using semi-permanent needles (42.9\%) and electroauriculotherapy (21.4\%). Conclusions: auriculotherapy was effective in reducing chronic musculoskeletal pain, showing itself as an alternative to be used for the promotion and recovery of individuals' health.

Descriptors: Auriculotherapy; Musculoskeletal Pain; Chronic Pain; Nursing; Review.

\section{RESUMO}

Objetivos: descrever as evidências científicas sobre o uso da auriculoterapia na redução da dor musculoesquelética crônica em adultos e idosos. Métodos: revisão integrativa de literatura realizada nas bases de dados Literatura Latino-Americana e do Caribe em Ciências da Saúde, SciVerse Scopus e MEDLINE (via National Library of Medicine), em março de 2019, sem recorte temporal. Resultados: foram analisados 14 artigos científicos originais, sendo $64,3 \%$ classificados com nível de evidência 2. Todas as produções mostram o benefício da auriculoterapia na redução da dor musculoesquelética crônica, em especial na região da coluna lombar (42,9\%). O tratamento foi desenvolvido em uma ou mais sessões, pelo uso de agulhas semipermanentes $(42,9 \%)$ e eletroauriculoterapia $(21,4 \%)$. Conclusões: a auriculoterapia foi eficaz na redução da dor musculoesquelética crônica, mostrando-se como uma alternativa a ser utilizada para a promoção e recuperação da saúde dos indivíduos.

Descritores: Auriculoterapia; Dor Musculoesquelética; Dor Crônica; Enfermagem; Revisão.

\section{RESUMEN}

Objetivos: describir las evidencias científicas sobre el uso de la auriculoterapia en la reducción del dolor musculoesquelético crónico en adultos y ancianos. Métodos: revisión integrativa de literatura realizada en las bases de datos Literatura Latinoamericana y de Caribe en Ciencias de la Salud, SciVerse Scopus y MEDLINE (via National Library of Medicine), en marzo de 2019, sin recorte temporal. Resultados: han sido analizados 14 artículos científicos originales, siendo $64,3 \%$ clasificados con nivel de evidencia 2. Todas las producciones muestran el beneficio de la auriculoterapia en la reducción del dolor musculoesquelético crónico, en especial en la región de la columna lumbar $(42,9 \%)$. El tratamiento ha sido desarrollado en una o más sesiones, por el uso de agujas semipermanentes $(42,9 \%)$ y electroauriculoterapia $(21,4 \%)$ Conclusiones: la auriculoterapia ha sido eficaz en la reducción del dolor musculoesquelético crónico, mostrándose como una alternativa a ser utilizada para la promoción y recuperación de la salud de los indivíduos.

Descriptores: Auriculoterapia; Dolor Musculoesquelético; Dolor Crónico; Enfermaría; Revisión. 


\section{INTRODUCTION}

When considering the musculoskeletal system, the scientific literature has shown high rates of painful symptoms in the Brazilian population, especially in adults ${ }^{(1-3)}$. The presence of symptoms such as musculoskeletal pain (MSP), tingling and paresthesia is considered as a warning sign to individuals, as it may indicate some biological change ${ }^{(4)}$.

In this scenario, MSP results from repetitive effort, overuse and work-related musculoskeletal disorders ${ }^{(5)}$. It is classified as acute or chronic. In particular, the latter is defined as persistent or recurrent, that is, it persists beyond the normal time for tissue healing and has a minimum duration of three months ${ }^{(6)}$. The consequences of chronic MSP represent negative, personal and economic impacts ${ }^{(7)}$. These include physical disability, reduced functionality and productivity, as well as temporary or permanent leave from work $^{(8)}$.

The treatment of chronic musculoskeletal symptoms can be through pharmacological resources or not, or with the combination of both. Among the non-pharmacological therapeutic resources, integrative and complementary practices (ICPs) stand out. They involve natural stimuli that promote disease prevention, health promotion and recovery. In Brazil, these practices were incorporated into Health Care in the 1980s, through the creation of the Unified Health System, in order to promote the inclusion of new health strategies and practices ${ }^{(9)}$.

However, only in 2006, ICPs were made official in Health Care through the National Policy of Integrative and Complementary Practices. At that time, they were constituted by five practices: Traditional Chinese Medicine (acupuncture, homeopathy, medicinal plants, phytotherapy and thermalism), crenotherapy and anthroposophical medicine ${ }^{(10)}$. Subsequently, in 2018, the Unified Health System started to have 29 ICPs available for access by the population, including auriculotherapy.

Auriculotherapy or auricular acupuncture uses reflex points in the outer ear to treat symptoms and diseases. As a mechanism of action, the pinna presents an abundant innervation composed of the auriculotemporal nerves, auricular branch of the vagus nerve, minor occipital and major auricular. They are responsible for electrical stimuli through the Alpha, Beta and Gamma fibers, for the stimulation of certain points, which will transmit information to nervous system structures such as: cranial nerves, limbic system, thalamus, hypothalamus, reticular formation, cerebellum and cerebral cortex ${ }^{(11)}$.

This practice is based on the precepts of Traditional Chinese Medicine, is easy to apply, uses non-invasive materials and has minimal side effects ${ }^{(12)}$. The national and international scientific literature has shown positive results in the treatment of several diseases, both psychic and physical ${ }^{(12-15)}$, showing itself, in this sense, as a promising practice ${ }^{(11)}$.

Given the above, the interface with the field of nursing and its commitment to the health of people in all instances of their lives stands out. In addition, the practice of auriculotherapy can be developed by qualified nurses, according to Resolutions $n^{\circ}$ $581 / 2018$ and $n^{\circ} 585 / 2018$ of the Federal Nursing Council(16-17), which regulate ICPs and acupuncture, respectively, as one of the nurse's specialties.

\section{OBJECTIVES}

To describe the scientific evidence on the use of auriculotherapy to reduce chronic MSP in adults and the elderly.

\section{METHODS}

It is an integrative literature review ${ }^{(18)}$. In carrying out this method, six steps were taken: identification of the theme and formulation of the research question; establishment of inclusion and exclusion criteria for articles; definition of the information to be extracted from the selected articles; evaluation; interpretation of results; and synthesis of evidenced knowledge ${ }^{(18)}$.

To elaborate the research question, the PICO strategy was used, in which adults and / or the elderly were adopted as P (population); I (intervention), auriculotherapy; and $\mathrm{O}$ (outcome), the reduction of chronic MSP (Figure 1) ${ }^{(19)}$. In this integrative review, the third element, that is, the comparison (C), was not used. Thus, the review question was formulated: "What is the scientific evidence about auriculotherapy in reducing chronic MSP in adults and the elderly?"

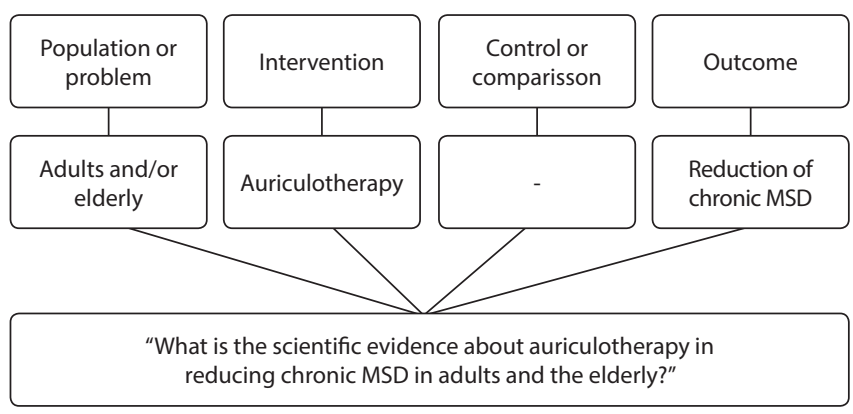

Note: MSP (musculoskeletal pain).

Figure 1 - Description of the PICO, Brazil strategy, 2019

The selection of articles was carried out in March 2019, in the Latin American and Caribbean Health Sciences (LILACS), SciVerse Scopus (Scopus) and MEDLINE databases (via National Library of Medicine - PubMed). For the search, the following inclusion criteria were considered: original articles (from primary studies), published in Portuguese, English or Spanish, available in electronic support online and that answered the review question. Exclusion criteria: articles developed with participants who were under 18 years old. There was no time cut. Duplicate articles were considered only once. Chronic MSP was defined as chronic MSP lasting more than three months, according to the classification of the Brazilian Society for the Study of Pain ${ }^{(20)}$.

From this, the search strategy was formulated in the LILACS database ("auriculotherapy"OR "auricular acupuncture") AND ("chronic pain" OR "low back pain" OR "cervicalgia" OR "cumulative traumatic disorders"); MEDLINE (via PubMed) $(((()(((($ "musculoskeletal pain" [MeSH Terms]) OR "chronic pain" [MeSH Terms]) OR "low back pain" [MeSH Terms]) OR"neck pain" [MeSH Terms]) OR ("musculoskeletal pain" OR "chronic pain" OR "low back pain" OR "neck pain"))) AND (("auriculotherapy"[MeSHTerms]) OR ("auriculotherapy"OR"auricular acupuncture")); and, in Scopus TITLE-ABS-KEY ((“"'musculoskeletal pain" OR "chronic pain" OR "low back pain "OR" neck pain") AND ("auriculotherapy"OR"auricular acupuncture"OR"auricular therapy")).

The productions were exported to the Endnote software, in order to assist in the selection of studies. First, the titles and 
abstracts of all items that responded to the primary search were read. Subsequently, the productions were read in full. The study selection process was carried out by two reviewers independently. When in a situation of divergence, a consensus was sought with the support of a third reviewer. This phase is represented in Figure 2.

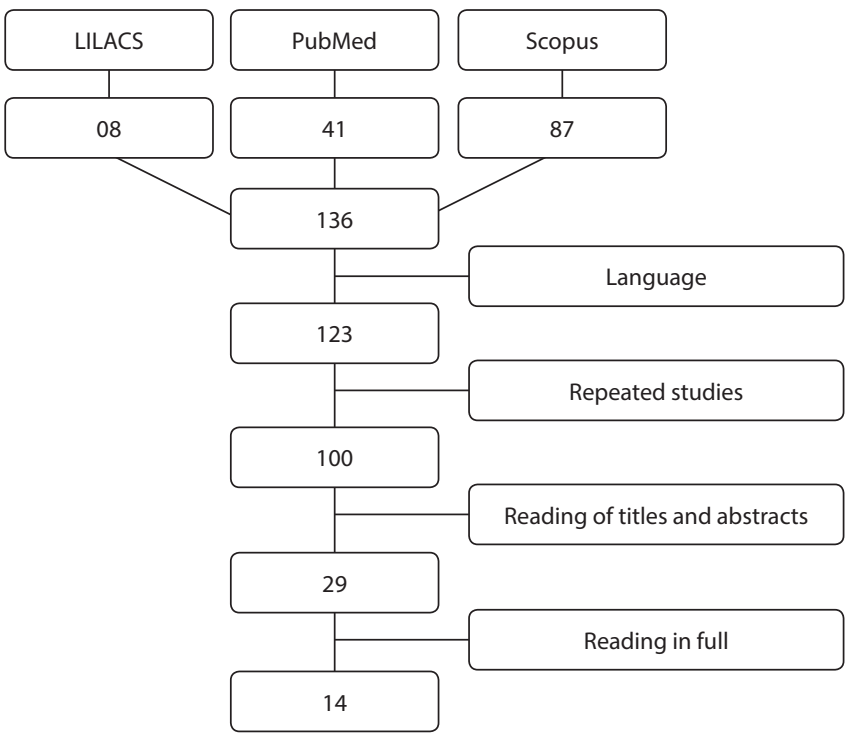

Figure 2 - Flow of the selection of studies included in the corpus of the integrative review, from the databases of Latin American and Caribbean Literature in Health Sciences, SciVerse Scopus and National Library of Medicine, Brazil, 2019
For the categorization phase of the studies, a synoptic table was developed, built in the text editor Microsoft Word 2010, containing variables related to the characterization of the articles (methodological design, level of evidence, type / location of MSP, participants and duration, ear points, material used [intervention and control, number of sessions], evaluation and main results).

The level of evidence of primary studies was also classified according to the type of clinical question in the studies, which may be: of significance; prognosis, prediction or etiology; and intervention, treatment or diagnostic / diagnostic test ${ }^{(21)}$.

\section{RESULTS}

With the studies in full, they were characterized according to methodological design, level of evidence, type/ location of MSP, participants and duration, ear points, material used (intervention and control, number of sessions), evaluation and results. It is shown in Chart 1.

Of the 14 studies included in this review, Brazil is highlighted as the country that most published and conducted the most research on the subject in question ( $N=5 ; 35.7 \%)$, specifically in the states of Paraná(22), São Paulo ${ }^{(23,28,30)}$ and Minas Gerais( ${ }^{(33)}$. Next, there are China ( $N=4 ; 28.6 \%)$, Austria ( $N=2 ; 14.3 \%)$, Germany $(\mathrm{N}=2 ; 14.3 \%)$ and the United States $(\mathrm{N}=1 ; 7.1 \%)$. As for the place of the study, physiotherapy clinics and / or rehabilitation center ${ }^{(22,24,33)}$, long-term care institution for the elderly ${ }^{(31-32)}$, university $^{(26-28)}$ and military region ${ }^{(34)}$. The others did not describe the study location ${ }^{(14,23,25,29-30)}$.

Chart 1 - Characterization of the articles analyzed in the integrative review, Brazil, 2019 ( $N=14)$

\begin{tabular}{|c|c|c|c|c|c|c|}
\hline Title & $\begin{array}{l}\text { Design, LE } \\
\text { and year of } \\
\text { publication }\end{array}$ & $\begin{array}{l}\text { Type / } \\
\text { location of } \\
\text { pain }\end{array}$ & $\begin{array}{l}\text { Participants } \\
\text { and duration }\end{array}$ & Auricular points & $\begin{array}{l}\text { Intervention and } \\
\text { control }\end{array}$ & Evaluation and result \\
\hline $\begin{array}{l}\text { Auriculotherapy in the } \\
\text { treatment of individuals } \\
\text { affected by work- } \\
\text { related musculoskeletal } \\
\text { disorders (wrmd) / } \\
\text { repetitive strain injuries } \\
\text { (RSI) }\end{array}$ & $\begin{array}{l}\text { Before and after } \\
\text { clinical trial } \\
\qquad \mathrm{LE}=3 \\
\text { Year: } 2006\end{array}$ & $\begin{array}{l}\text { Work-related } \\
\text { diseases } \\
\text { (WRD) }\end{array}$ & $\begin{array}{l}\text { Participants: } 12 \\
\text { (workers) } \\
\text { Duration: Ten } \\
\text { sessions (twice a } \\
\text { week) }\end{array}$ & $\begin{array}{l}\text { Apex of the ear, } \\
\text { shen men, kidney, } \\
\text { sympathetic, } \\
\text { liver, spleen- } \\
\text { pancreas, points } \\
\text { related to painful } \\
\text { areas }\end{array}$ & Rapeseed seeds & $\begin{array}{l}\text { Evaluation: Visual Analog } \\
\text { Scale } \\
\text { Result: reduction of pain } \\
(p<0.001)\end{array}$ \\
\hline $\begin{array}{l}\text { Evaluation of atrial } \\
\text { acupressure in painful } \\
\text { shoulder syndrome: a } \\
\text { case study }{ }^{(23)}\end{array}$ & $\begin{array}{l}\text { Case study } \\
\qquad L E=6 \\
\text { Year: } 2013\end{array}$ & $\begin{array}{l}\text { Painful } \\
\text { shoulder } \\
\text { syndrome }\end{array}$ & $\begin{array}{l}\text { Participants: } 1 \\
\text { (military police) } \\
\text { Duration: } 12 \\
\text { sessions (weekly) }\end{array}$ & $\begin{array}{l}\text { Shen men, kidney, } \\
\text { sympathetic, } \\
\text { shoulder, } \\
\text { shoulder joint } \\
\text { and clavicle }\end{array}$ & Crystal spheres & $\begin{array}{l}\text { Evaluation: Penn Shoulder } \\
\text { Score } \\
\text { Result: pain reduction and } \\
\text { sleep improvement }\end{array}$ \\
\hline $\begin{array}{l}\text { Exercise and Auricular } \\
\text { Acupuncture for } \\
\text { Chronic Low-back } \\
\text { Pain: A Feasibility } \\
\text { Randomized- } \\
\text { Controlled Trial (24) }\end{array}$ & $\begin{array}{l}\text { Randomized } \\
\text { clinical trial } \\
\text { LE }=2 \\
\text { Year: } 2012\end{array}$ & Lower back & $\begin{array}{l}\text { Participants: } 51 \\
\text { (students) } \\
\text { Duration: } 12 \\
\text { weeks (6 face- } \\
\text { to-face and } \\
6 \text { telephone } \\
\text { support) }\end{array}$ & $\begin{array}{l}\text { Shen men, } \\
\text { lumbar spine } \\
\text { and cushion }\end{array}$ & $\begin{array}{l}\text { Intervention: } \\
\text { auriculotherapy } \\
\text { (semi-permanent } \\
\text { needles) and } \\
\text { physical exercise } \\
\text { Control: physical } \\
\text { exercise }\end{array}$ & $\begin{array}{l}\text { Evaluation: Oswestry } \\
\text { Disability Questionnaire; } \\
\text { Analogic visual scale } \\
\text { Result: reduction of } \\
\text { pain; and use of pain } \\
\text { relief medication in } \\
\text { the intervention group } \\
(73.9 \%) \text { compared to } \\
\text { control }(41.2 \%)\end{array}$ \\
\hline $\begin{array}{l}\text { Multimodal Care in } \\
\text { the Management of a } \\
\text { Patient with Chronic } \\
\text { Tendinopathy of the } \\
\text { Biceps Femoris: a Case } \\
\text { Report }^{(25)}\end{array}$ & $\begin{array}{l}\text { Case study } \\
\qquad \text { LE }=6 \\
\text { Year: } 2017\end{array}$ & $\begin{array}{l}\text { Right } \\
\text { femoral } \\
\text { muscle }\end{array}$ & $\begin{array}{l}\text { Participants: } 1 \\
\text { (patient) } \\
\text { Duration: Eight } \\
\text { sessions (twice a } \\
\text { week) and three } \\
\text { sessions (weekly) }\end{array}$ & $\begin{array}{l}\text { Apex of the } \\
\text { ear, shen men, } \\
\text { sympathetic, } \\
\text { liver, spleen, } \\
\text { kidney, liver } \\
\text { yang, hip }\end{array}$ & $\begin{array}{l}\text { Auriculotherapy } \\
\text { combined with dry } \\
\text { needling }\end{array}$ & $\begin{array}{l}\text { Evaluation: Numerical Pain } \\
\text { Scale } \\
\text { Result: reduction of pain, } \\
\text { fatigue, symptoms of } \\
\text { anxiety and tension; and } \\
\text { improving sleep quality }\end{array}$ \\
\hline
\end{tabular}


Chart 1

\begin{tabular}{|c|c|c|c|c|c|c|}
\hline Title & $\begin{array}{l}\text { Design, LE } \\
\text { and year of } \\
\text { publication }\end{array}$ & $\begin{array}{c}\text { Type / } \\
\text { location of } \\
\text { pain }\end{array}$ & $\begin{array}{l}\text { Participants } \\
\text { and duration }\end{array}$ & Auricular points & $\begin{array}{l}\text { Intervention and } \\
\text { control }\end{array}$ & Evaluation and result \\
\hline $\begin{array}{l}\text { Electrical Stimulation } \\
\text { of Auricular } \\
\text { Acupuncture Points } \\
\text { Is More Effective Than } \\
\text { Conventional Manual } \\
\text { Auricular Acupuncture } \\
\text { in Chronic Cervical } \\
\text { Pain: A Pilot Study }\end{array}$ & $\begin{array}{l}\text { Randomized } \\
\text { clinical trial } \\
\text { LE }=2 \\
\text { Year: } 2003\end{array}$ & Cervical & $\begin{array}{l}\text { Participants: } 21 \\
\text { (patients) } \\
\text { Duration: Six } \\
\text { sessions (weekly) }\end{array}$ & $\begin{array}{l}\text { Shen men, } \\
\text { cervical spine } \\
\text { and cushion }\end{array}$ & $\begin{array}{l}\text { Intervention: } \\
\text { electroacupuncture } \\
\text { Control: } \\
\text { auriculotherapy } \\
\text { (semi-permanent } \\
\text { needles) }\end{array}$ & $\begin{array}{l}\text { Evaluation: Visual Analog } \\
\text { Scale } \\
\text { Result: greater reduction } \\
\text { in chronic cervical pain } \\
\text { and medication use; } \\
\text { improvement in sleep } \\
\text { quality and psychological } \\
\text { well-being in the } \\
\text { intervention group, when } \\
\text { compared to control (p } \\
<0.05 \text { ) }\end{array}$ \\
\hline $\begin{array}{l}\text { The Short- and } \\
\text { Long-Term Benefit in } \\
\text { Chronic Low Back Pain } \\
\text { Through Adjuvant } \\
\text { Electrical Versus } \\
\text { Manual Auricular } \\
\text { Acupuncture }^{(27)}\end{array}$ & $\begin{array}{l}\text { Randomized } \\
\text { clinical trial } \\
\text { LE }=2 \\
\text { Year: } 2004\end{array}$ & Lower back & $\begin{array}{l}\text { Participants: } 61 \\
\text { (patients) } \\
\text { Duration: Six } \\
\text { sessions (weekly) }\end{array}$ & $\begin{array}{l}\text { Shen men, } \\
\text { lumbar spine } \\
\text { and cushion }\end{array}$ & $\begin{array}{l}\text { Intervention: } \\
\text { electroacupuncture } \\
\text { Control: } \\
\text { auriculotherapy } \\
\text { (semi-permanent } \\
\text { needles) }\end{array}$ & $\begin{array}{l}\text { Evaluation: Visual Analog } \\
\text { Scale } \\
\text { Result: greater pain } \\
\text { reduction in the } \\
\text { intervention group, when } \\
\text { compared to control ( } p< \\
0.001) \text { and medication use } \\
\text { ( } p<0.001) \text {; improvement } \\
\text { in psychological well- } \\
\text { being and sleep }\end{array}$ \\
\hline $\begin{array}{l}\text { Effect of a single } \\
\text { session of ear } \\
\text { acupuncture on pain } \\
\text { intensity and postural } \\
\text { control in individuals } \\
\text { with chronic low back } \\
\text { pain: a randomized } \\
\text { controlled trial }\end{array}$ & $\begin{array}{l}\text { Randomized } \\
\text { clinical trial } \\
\text { LE }=2 \\
\text { Year: } 2016\end{array}$ & Lower back & $\begin{array}{l}\text { Participants: } 80 \\
\text { (patients) } \\
\text { Duration: One } \\
\text { session }\end{array}$ & $\begin{array}{l}\text { Shen men, } \\
\text { lumbar spine } \\
\text { and cushion }\end{array}$ & $\begin{array}{l}\text { Intervention: } \\
\text { auriculotherapy } \\
\text { (semipermaning } \\
\text { needles) } \\
\text { Control: ultrasound } \\
\text { on but not } \\
\text { activated }\end{array}$ & $\begin{array}{l}\text { Evaluation: Numerical Pain } \\
\text { Scale } \\
\text { Result: greater pain } \\
\text { reduction in the } \\
\text { intervention group when } \\
\text { compared to the control } \\
\text { group ( } p=0.032)\end{array}$ \\
\hline $\begin{array}{l}\text { Auricular therapy } \\
\text { for chronic pain } \\
\text { management in } \\
\text { adults: A synthesis of } \\
\text { evidence } \\
\text { evi) }\end{array}$ & $\begin{array}{l}\text { Systematic } \\
\text { review and } \\
\text { meta-analysis } \\
\qquad \mathrm{LE}=1 \\
\text { Year: } 2015\end{array}$ & $\begin{array}{l}\text { Chronic } \\
\text { pains }\end{array}$ & $\begin{array}{l}\text { Participants: } 12 \\
\text { to } 265 \\
\text { (patients) } \\
\text { Duration: One to } \\
12 \text { weeks }\end{array}$ & $\begin{array}{l}\text { Most used: shen } \\
\text { men }\end{array}$ & $\begin{array}{l}\text { Vaccaria seeds } \\
\text { and semi- } \\
\text { permanent needles } \\
\text { (electrostimulation) }\end{array}$ & $\begin{array}{l}\text { Evaluation: Visual } \\
\text { Analogue Scale; Numerical } \\
\text { Pain Scale } \\
\text { Result: reduced intensity } \\
\text { of chronic low back pain } \\
\text { and tension headaches }\end{array}$ \\
\hline $\begin{array}{l}\text { Efficacy of Auricular } \\
\text { Acupressure for } \\
\text { Chronic Low Back Pain: } \\
\text { A Systematic Review } \\
\text { and Meta-Analysis } \\
\text { of Randomized } \\
\text { Controlled Trials }\end{array}$ & $\begin{array}{l}\text { Systematic } \\
\text { review and } \\
\text { meta-analysis } \\
\qquad \text { LE }=1 \\
\text { Year: } 2017\end{array}$ & Lower back & $\begin{array}{l}\text { Participants: } 19 \\
\text { to } 74 \\
\text { (adult patients) } \\
\text { Duration: Two to } \\
12 \text { weeks }\end{array}$ & $\begin{array}{l}\text { Most used: shen } \\
\text { men, subcortex, } \\
\text { lumbosacral } \\
\text { region, liver, } \\
\text { kidney and } \\
\text { sympathetic }\end{array}$ & $\begin{array}{l}\text { Vaccaria seeds and } \\
\text { metal spheres }\end{array}$ & $\begin{array}{l}\text { Evaluation: Visual Analog } \\
\text { Scale, Verbal Rating Scale, } \\
\text { Brief Pain Inventory Short } \\
\text { Form } \\
\text { Result: pain reduction ( } p \\
<0.001 \text { ) }\end{array}$ \\
\hline $\begin{array}{l}\text { Evaluation of the } \\
\text { immediate effect of } \\
\text { auricular acupuncture } \\
\text { on pain and } \\
\text { electromyographic } \\
\text { activity of the upper } \\
\text { trapezius muscle } \\
\text { in patients with } \\
\text { nonspecific neck } \\
\text { pain: A randomized, } \\
\text { single-blinded, sham- } \\
\text { controlled, crossover } \\
\text { study }^{(30)}\end{array}$ & $\begin{array}{l}\begin{array}{c}\text { Randomized } \\
\text { clinical trial }\end{array} \\
\text { LE }=2 \\
\text { Year: } 2015\end{array}$ & $\begin{array}{l}\text { Músculo } \\
\text { trapézio e } \\
\text { cervicalgia } \\
\text { unspecific }\end{array}$ & $\begin{array}{l}\text { Participantes: } 24 \\
\text { (pacientes) } \\
\text { Duration: One } \\
\text { session }\end{array}$ & $\begin{array}{l}\text { Grupo- } \\
\text { intervenção: } \\
\text { Cintura escapular } \\
\text { e ombro } \\
\text { Placebo: regions } \\
\text { that were } \\
\text { unrelated to the } \\
\text { outcome }\end{array}$ & $\begin{array}{l}\text { Intervenção: } \\
\text { agulhas } \\
\text { semipermanentes } \\
\text { Placebo: semi- } \\
\text { permanent needles }\end{array}$ & $\begin{array}{l}\text { Avaliação: Escala Numérica } \\
\text { da Dor } \\
\text { Result: pain reduction ( } p \\
<0.001 \text { ) in both groups }\end{array}$ \\
\hline $\begin{array}{l}\text { Longitudinal changes } \\
\text { in the disability level } \\
\text { of the elders with } \\
\text { low back pain after } \\
\text { auriculotherapy }{ }^{(31)}\end{array}$ & $\begin{array}{l}\text { Randomized } \\
\text { controlled } \\
\text { clinical trial } \\
\text { LE }=2 \\
\text { Year: } 2008\end{array}$ & Backache & $\begin{array}{l}\text { Participants: } 60 \\
\text { (elderly patients) } \\
\text { Duration: Three } \\
\text { sessions } \\
\text { (weekly) }\end{array}$ & $\begin{array}{l}\text { Shen men, } \\
\text { kidney, } \\
\text { bladder, region, } \\
\text { lumbosacral } \\
\text { region, liver, } \\
\text { spleen and } \\
\text { coccyx }\end{array}$ & $\begin{array}{l}\text { Intervention: } \\
\text { magnetic spheres } \\
\text { Control: vaccaria } \\
\text { seed }\end{array}$ & $\begin{array}{l}\text { Evaluation: Aberdeen low } \\
\text { back pain disability scale } \\
\text { Result: the intervention } \\
\text { group showed greater } \\
\text { pain reduction, improved } \\
\text { physical and functional } \\
\text { capacity and abilities ( } p \\
<0.001 \text { ) when compared } \\
\text { to the control group }\end{array}$ \\
\hline
\end{tabular}

To be continued 


\begin{tabular}{|c|c|c|c|c|c|c|}
\hline Title & $\begin{array}{l}\text { Design, LE } \\
\text { and year of } \\
\text { publication }\end{array}$ & $\begin{array}{c}\text { Type / } \\
\text { location of } \\
\text { pain }\end{array}$ & $\begin{array}{l}\text { Participants } \\
\text { and duration }\end{array}$ & Auricular points & $\begin{array}{l}\text { Intervention and } \\
\text { control }\end{array}$ & Evaluation and result \\
\hline $\begin{array}{l}\text { Auriculotherapy on } \\
\text { low back pain in the } \\
\text { elderly }\end{array}$ & $\begin{array}{l}\text { Randomized } \\
\text { controlled } \\
\text { clinical trial } \\
\text { LE }=2 \\
\text { Year: } 2007\end{array}$ & Backache & $\begin{array}{l}\text { Participants: } 60 \\
\text { (elderly patients) } \\
\text { Duration: Three } \\
\text { sessions } \\
\text { (weekly) }\end{array}$ & $\begin{array}{l}\text { Shen men, } \\
\text { kidney, bladder, } \\
\text { lumbosacral } \\
\text { region, liver, } \\
\text { spleen and } \\
\text { coccyx }\end{array}$ & $\begin{array}{l}\text { Intervention: } \\
\text { magnetic spheres } \\
\text { Control: vaccaria } \\
\text { seed }\end{array}$ & $\begin{array}{l}\text { Rating: Chinese Pain } \\
\text { Intensity Verbal Rating } \\
\text { Scale } \\
\text { Result: the intervention } \\
\text { group showed greater } \\
\text { pain reduction when } \\
\text { compared to the control } \\
\text { group }(p<0.001)\end{array}$ \\
\hline $\begin{array}{l}\text { Effects of auricular } \\
\text { acunpucture on chonic } \\
\text { pain in people with } \\
\text { back musculoskeletal } \\
\text { disorders: a } \\
\text { randomized clinical } \\
\text { trial( }^{(33)}\end{array}$ & $\begin{array}{c}\text { Randomized } \\
\text { clinical trial } \\
\text { LE }=2 \\
\text { Year: } 2019\end{array}$ & $\begin{array}{l}\text { Pain in the } \\
\text { spine }\end{array}$ & $\begin{array}{l}\text { Participants: } \\
110 \text { (adults and } \\
\text { the elderly) } \\
\text { Duration: Five } \\
\text { sessions (weekly) }\end{array}$ & $\begin{array}{l}\text { Intervention } \\
\text { group: Shen } \\
\text { men, kidney, } \\
\text { sympathetic } \\
\text { nerve, points of } \\
\text { restoring energy } \\
\text { balance, cervical, } \\
\text { thoracic and / or } \\
\text { lumbar vertebrae } \\
\text { Placebo group: } \\
\text { Eye point }\end{array}$ & $\begin{array}{l}\text { Intervention and } \\
\text { placebo: semi- } \\
\text { permanent needles } \\
\text { Control: received no } \\
\text { intervention }\end{array}$ & $\begin{array}{l}\text { Evaluation: Brief Inventory } \\
\text { of Pain; digital algometry } \\
\text { Result: there was a } \\
\text { reduction in pain intensity } \\
\text { in the intervention and } \\
\text { placebo group between } \\
\text { the initial and final } \\
\text { evaluation }(p<0.05) \text {, and } \\
\text { in the intervention group } \\
\text { between the initial and } \\
\text { follow-up evaluation ( } p \\
<0.05 \text { ) }\end{array}$ \\
\hline $\begin{array}{l}\text { Auricular Acupuncture } \\
\text { for Chronic Pain } \\
\text { and Insomnia: A } \\
\text { Randomized Clinical } \\
\text { Trial }{ }^{(34)}\end{array}$ & $\begin{array}{l}\text { Randomized } \\
\text { clinical trial } \\
\text { LE }=2 \\
\text { Year: } 2018\end{array}$ & $\begin{array}{l}\text { Chronic } \\
\text { pains }\end{array}$ & $\begin{array}{l}\text { Participants: } 45 \\
\text { active and / or } \\
\text { retired military } \\
\text { personnel } \\
\text { Duration: } 1 \\
\text { session }\end{array}$ & $\begin{array}{l}\text { Cingulate Gyrus, } \\
\text { Thalamus } \\
\text { point, Omega 2, } \\
\text { Point Zero and } \\
\text { Shenmen }\end{array}$ & $\begin{array}{l}\text { Intervention: semi- } \\
\text { permanent needle } \\
\text { Control: actigraph } \\
\text { device (non- } \\
\text { invasive method to } \\
\text { monitor human rest } \\
\text { / activity cycles) }\end{array}$ & $\begin{array}{l}\text { Assessment: Brief } \\
\text { Inventory of Pain } \\
\text { Result: the intervention } \\
\text { group showed greater } \\
\text { reduction in pain ( } p \\
=0.001) \text {, insomnia ( } p \\
=0.018 \text { ) and better } \\
\text { satisfaction with life }(p= \\
0.007 \text { ) when compared to } \\
\text { the control group }\end{array}$ \\
\hline
\end{tabular}

In relation to the academic background of the authors, Phys-

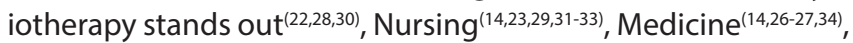
Health Sciences and Rehabilitation ${ }^{(24)}$, Basic and Clinical Sciences ${ }^{(25)}$ and Epidemiology ${ }^{(14)}$. With regard to the year of publication of the studies, there has been an increase in scientific production in the last five years (2015 to 2019), which points to a relatively current production.

With regard to methodological designs, clinical trials stood out: randomized $(\mathrm{N}=7 ; 50 \%)$ and randomized controlled trials $(\mathrm{N}=2 ; 14.3 \%$,). In addition, there was a case study $(\mathrm{N}=2 ; 14.3 \%)$, systematic review and meta-analysis ( $\mathrm{N}=2 ; 14.3 \%)$ and of the type before and after $(\mathrm{N}=1 ; 7.1 \%)$.

As for the levels of evidence (Figure 3), all studies presented the type of clinical question aimed at intervention or treatment, being classified as level $2(\mathrm{~N}=9 ; 64.3 \%)$.

There was a predominance of productions that researched auriculotherapy with patients $(\mathrm{N}=9 ; 64.2 \%)$ who reported chronic MSP(14,25-30,32-33). Auriculotherapy treatment was applied to adults and / or the elderly, especially in the lumbar spine ( $N=6 ; 42.9 \%)$ $(14,24,27-28,3132)$. For treatment, the most used ear points were those related to the region of pain and the Shen men $(N=13 ; 92.9 \%)$.

It was observed that there was a reduction in chronic MSP between the first and the last application of auriculotherapy (Chart 2). Different methods and materials were used for application. With regard to the electroacupuncture technique $(\mathrm{N}=3$; $21.4 \%$ ), it was evidenced that this presented significant results ( $p$
$<0.05)$ in terms of the reduction of chronic pain in the cervical and lumbar region, in comparison with the use of semi-permanent needles for 48 hours $^{(26-27)}$.

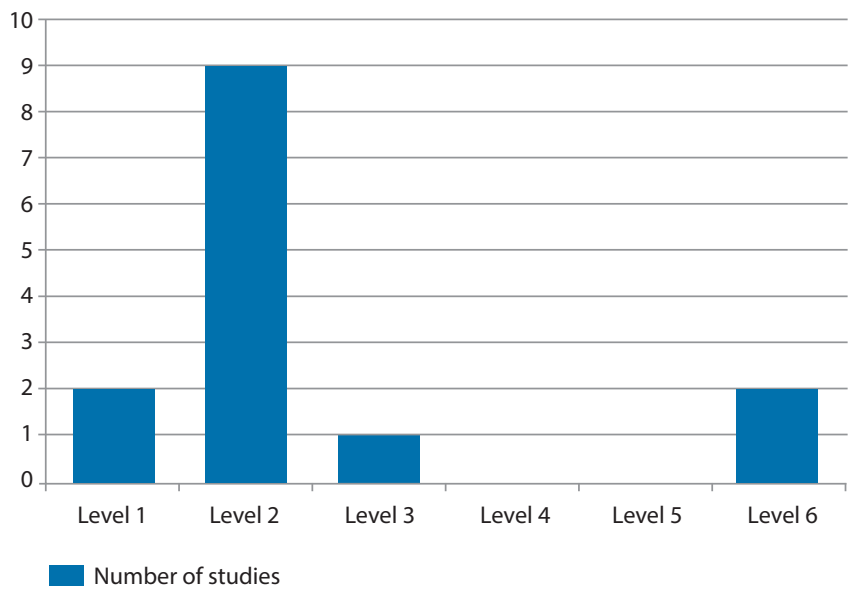

Figure 3 - Distribution of articles regarding the level of evidence ${ }^{(21)}$, Brazil, $2019(\mathrm{~N}=14)$

However, studies have found that auricular therapy performed using semi-permanent needles $(\mathrm{N}=6 ; 42.9)$, metallic spheres $(\mathrm{N}=$ $2 ; 14.3 \%)$ and seeds $(N=1 ; 7.1 \%)$ also obtained positive results in the reduction of chronic MSP(22-24,26,30-31,33-34). In addition, it has been shown that auriculotherapy can have a lasting effect on the relief of chronic MSP within one month after the end of treatment ${ }^{(29)}$. 
As for the reduction of the intensity of chronic MSP, the use of auriculotherapy as a therapy was favorable in the studies, as shown in Chart 2. A drop in pain intensity scores stands out, especially in those who used the Visual Analogue Scale, with a maximum reduction of 5 points ${ }^{(22,25)}$. Reductions were also observed with percentages of $10.6 \%$ and $46.7 \%$ on the intensity of this chronic symptomatology ${ }^{(14,23-24,28-29)}$. However, there are studies that did not provide quantitative data on the reduction of pain intensity, which made it impossible to include them in Chart 2.

Chart 2 - Pain intensity before and after the intervention, according to the instruments used, Brazil, 2019

\begin{tabular}{|c|c|c|c|c|}
\hline \multirow{2}{*}{$\begin{array}{l}\text { Evaluation } \\
\text { tool }\end{array}$} & \multirow{2}{*}{ Groups } & \multicolumn{2}{|c|}{ Pain intensity* } & \multirow{2}{*}{$\begin{array}{l}\text { Number of } \\
\text { sessions }\end{array}$} \\
\hline & & Before & After & \\
\hline $\begin{array}{c}\text { Analogic visual } \\
\text { scale }^{(22)}\end{array}$ & - & $5.86( \pm 2.23)$ & $1.19( \pm 1.18)$ & 10 \\
\hline PSS-Brazil ${ }^{(23)}$ & - & $0.0 \%^{\dagger}$ & $46.7 \%^{\ddagger}$ & 12 \\
\hline $\begin{array}{l}\text { Analogic visual } \\
\text { scale }^{(25)}\end{array}$ & - & 6 & 1 & 19 \\
\hline \multirow{2}{*}{$\begin{array}{l}\text { Numerical Pain } \\
\text { Scale }^{(30)}\end{array}$} & Intervention & $4,25( \pm 1,13)$ & $2,25( \pm 0,97)$ & \multirow{2}{*}{4} \\
\hline & Placebo & $4,00( \pm 0,73)$ & $2,33( \pm 1,07)$ & \\
\hline \multirow{2}{*}{$\begin{array}{l}\text { Chinese Pain } \\
\text { Intensity } \\
\text { Verbal Rating } \\
\text { Scale }^{(32)}\end{array}$} & Intervention & $2.73( \pm 0.74)$ & $1.87( \pm 0.68)$ & \multirow[b]{2}{*}{3} \\
\hline & Control & $2.47( \pm 0.78)$ & $2.27( \pm 0.58)$ & \\
\hline \multirow{3}{*}{$\begin{array}{l}\text { Brief Inventory } \\
\text { of Pain }\end{array}$} & Intervention & $4.86( \pm 2.79)$ & $2.46( \pm 3.03)$ & \multirow{3}{*}{5} \\
\hline & Placebo & $4.89( \pm 2.74)$ & $2.89( \pm 2.98)$ & \\
\hline & Control & $3.68( \pm 3.11)$ & $3.65( \pm 3.35)$ & \\
\hline \multirow{2}{*}{$\begin{array}{l}\text { Brief Inventory } \\
\text { of Pain }\end{array}$} & Intervention & $4.7( \pm 2.0)$ & $3,1( \pm 1.8)$ & \multirow{2}{*}{1} \\
\hline & Control & $3.6( \pm 1.7)$ & $3.6( \pm 2.3)$ & \\
\hline
\end{tabular}

Furthermore, in addition to the intensity of the pain, the evidence indicates that auriculotherapy proved to be favorable in helping to reduce pain, by relieving symptoms ${ }^{(14,24,2627,29,31)}$, physical disability ${ }^{(24,31)}$, aid in physical and functional skills ${ }^{(31)}$. There was also an improvement in the quality of sleep and aspects of wellbeing of individuals ${ }^{(23,26-27,31-32)}$, who, similarly, showed a reduction in the consumption of medications during the treatment period with auriculotherapy ${ }^{(24,26,34)}$.

\section{DISCUSSION}

The analyzed articles demonstrate that auriculotherapy is an effective technique to reduce chronic MSP and is well accepted by individuals. This evidence is of great relevance for assistance, considering that auriculotherapy is configured as a therapeutic resource of easy application and with a relative absence of side effects, providing assistance in health promotion and recovery ${ }^{(15)}$.

Chronic pain, according to the principles of Traditional Chinese Medicine, may result from blood stagnation, which will block the channels, that is, the meridians, causing a painful process and inefficiency in the activity of the organs ${ }^{(29,35)}$. From this perspective, it is believed that the auricular points and the meridians are associated with Zang Fu, that is, with the organs and viscera that correspond to the organism's functions ${ }^{(29)}$. It is noteworthy, therefore, that the therapeutic principle of Traditional Chinese Medicine is to relax the muscles and promote blood circulation, which, therefore, will activate the meridians and regulate the functions of the Zang Fu, providing pain control and relief ${ }^{(29,35)}$.

The most researched anatomical region for pain reduction, among the studies, was the lumbar spine. Pain in this region affects around $60 \%$ to $80 \%$ of the world population, and $20 \%$ of individuals progress to the development of chronic pain ${ }^{(29)}$. This is considered a complex and heterogeneous medical condition, as it presents a variety of symptoms and impacts on people's daily lives ${ }^{(36)}$. It can favor physical and functional incapacity, which are responsible for causing a compromise in the development of daily activities, as well as impairing the quality of sleep ${ }^{(37)}$.

The level of physical disability may vary according to the intensity and threshold of pain ${ }^{(15)}$. Therefore, by reducing the intensity of chronic pain through auriculotherapy, health and improved quality of life can be promoted. That is, there is rehabilitation for the development of daily activities, as well as improvement in sleep quality ${ }^{(15)}$.

Among the most used points for atrial stimulation, the shen men stand out, whose main purposes are to predispose the regions of the trunk and cerebral cortex to receive and decode the reflexes of the other regions to be stimulated later. This point is also related to the production of loads of natural hormones, such as endorphins, which will provide an aid in the relief of pain, as well as malaise ${ }^{(23)}$.

It was evidenced that the use of different materials to stimulate the auricular points was valid and presented positive results in the reduction of chronic MSP. In particular, electrical stimulation stands out, which showed a significant reduction in chronic MSP when compared to the use of semi-permanent needles. This may occur due to the use of electrical current at the ear points, which increases the effectiveness of analgesia at the site ${ }^{(26)}$, since, according to the intensity of the electrical stimulation, different types of endorphins are released ${ }^{(27)}$.

In addition, the use of semi-permanent needles, seeds and spheres also provided relief in the intensity of chronic MSP. This corroborates studies developed in Minas Gerais, which observed that the use of auriculotherapy by means of semi-permanent needles was effective in reducing chronic MSP in the spine region. It also helped in the clinical improvement of physical disability and in the development of daily activities ${ }^{(15,33)}$. Still, a meta-analysis found that the use of seeds and metallic spheres for the stimulation of reflex auricular points presented significant results in relation to the reduction of chronic pain in the low back ${ }^{(14)}$.

Semi-permanent needles, unlike seeds, do not need to be stimulated manually, which produces continuous stimulation through innervations in the auricular region until they are removed $^{(38)}$. However, the needles present greater discomfort during application and stay in the auricular points. In contrast, the use of seeds and metallic spheres is better accepted, as discomfort is minimal. However, these options require the participation of patients in the stimulation of points, which can be a limiting factor to scientific research ${ }^{(39)}$. 
As for the number of sessions offered, there was no pattern during treatment. This may occur due to the precepts of Traditional Chinese Medicine, in which individual treatments are designed according to the energy imbalance of each individual and their needs ${ }^{(39)}$.

\section{Study limitations}

As a limitation of the study, the difficulty in comparing the results related to the reduction of pain intensity, before and after intervention, is pointed out, since the studies do not present a standard in the presentation of results, nor of parameters or scales.

\section{Contributions to the field of Nursing}

This study brings contributions to the health field, nursing area and, above all, to the ICPs, so that services and professionals qualified to practice auriculotherapy can formulate intervention programs, for health problems, based on the demands pointed out by the scientific evidence. Therefore, the importance and effectiveness of this practice in health promotion and recovery is emphasized, especially in the treatment of chronic musculoskeletal symptoms.

\section{CONCLUSIONS}

The evidence from this study makes it possible to conclude that auriculotherapy contributed to the reduction of chronic MSP, especially in the lumbar spine region, developed in one or more sessions, by the use of electro-auriculotherapy. In practice, it has proven to be a safe technique, which provides both the relief of musculoskeletal symptoms and has beneficial effects on health and well-being.

\section{FUNDING}

The authors are grateful to the Programa de Bolsas de Iniciação Científica (PROBIC) - Fundação de Amparo à Pesquisa do Estado do Rio Grande do Sul (Fapergs) and Coordenação de Aperfeiçoamento de Pessoal de Nível Superior, Brasil (CAPES) (Finance Code 001) for providing scholarships and financial support for this research.

\section{REFERENCES}

1. Assunção AA, Abreu MNS. Factor associated with self-reported work-related musculoskeletal disorders in Brazilian adults. Rev Saude Publica. 2017;51(suppl 1):1-10s. doi: 10.1590/S1518-8787.2017051000282

2. Greco PBT, Magnago TSBS, Luz EMF, Ongaro JD, Chagas BP, Dullius AIS. Prevalência e fatores associados à ocorrência de dor musculoesquelética em agentes socioeducadores. Rev RENE. 2017;18(2):164-72. doi: 10.15253/2175-6783.2017000200004

3. Luz EMF, Magnago TSBS, Greco PBT, Dal Ongaro J, Lanes TC, Lemos JC. Prevalence and factors associated with musculoskeletal pain in hospital cleaning workers. Text \& context nursing. 2017;26(2):e00870016. doi: 10.1590/0104-07072017000870016

4. Morais BX, Magnago TSBS, Cauduro GM, Dalmolin GL, Pedro CMP, Gonçalves NGC. Fatores associados à dor musculoesquelética em estudantes de enfermagem. Rev Enferm UFSM. 2017;7(2):206-221. doi: 10.5902/2179769226442

5. Sociedade Brasileira para o Estudo da Dor. Ano mundial contra dor musculoesquelética. Dor Musculoesquelética. 2009. 2 p.

6. Perrot S, Cohen M, Barke A, Korwisi B, Rief W, Treede RD. The IASP classification of chronic pain for ICD-11: chronic secondary musculoskeletal pain. Pain. 2019;160(1):77-92. doi: 10.1097/j.pain.0000000000001389

7. Ribeiro RP, Sedrez JA, Candotti CT, Vieira A. Relationship between chronic non-specific low back pain with disability, static posture and flexibility. Fisioter Pesqui (Online). 2018;25(4):425-31. doi: 10.1590/1809-2950/18001925042018

8. Malta DC, Bernal RTI, Lima MG, Araújo SSC, Silva MMA, Freitas MIF, et al. Noncommunicable diseases and the use of health services: analysis of the National Health Survey in Brazil. Rev Saude Publica. 2017;51(suppl 1):1-4s. doi: 10.1590/s1518-8787.2017051000090

9. Dalmolin IS, Heidemann ITSB. Integrative and complementary practices and the interface with the health promotion: integrative review. Ciênc Cuid Saúde. 2017;16(3):1-8. doi: 10.4025/cienccuidsaude.v16i3.33035

10. Ministério da Saúde (BR). Secretaria de Atenção à Saúde. Política Nacional de Práticas Integrativas e complementares [Internet]. Brasília: Ministério da Saúde, 2006 [cited 2019 May 07]. Available from: http://bvsms.saude.gov.br/bvs/publicacoes/pnpic.pdf

11. Vieira A, Reis AM, Matos LC, Machado J, Moreira A. Does auriculotherapy have therapeutic effectiveness? an overview of systematic reviews. Complement Ther Clin Pract. 2018;33:61-70. doi: 10.1016/j.ctcp.2018.08.005

12. Prado JM, Kurebayashi LFS, Silva MJP. Experimental and placebo auricotherapy for stressed nurses: randomized controlled trial. Rev Esc Enferm USP. 2018;52:e03334. doi: 10.1590/s1980-220x2017030403334

13. Zhang ZX, Li CR, Rong PJ, Bai ZH, Hill AM, Jing Q, et al. Efficacy and safety of auricular therapy for depression. Med Acupunct. 2016;28(5):25667. doi: 10.1089/acu.2016.1182

14. Yang LH, Duan PB, Hou QM, Du SZ, Sun JF, Mei SJ, et al. Efficacy of auricular acupressure for chronic low back pain: a systematic review and meta-analysis of randomized controlled trials. Evid Based Complement Alternat Med. 2017.1-14. doi: 10.1155/2017/6383649

15. Moura CC, lunes DH, Ruginsk SG, Souza VHS, Assis BB, Chaves ECL. Action of ear acupunture in people with chronic pain the spinal column: a randomized clinical trial. Rev Latino-Am Enfermagem. 2018;26:e3050. doi: 10.1590/1518-8345.2678.3050

16. Conselho Federal de Enfermagem (COFEN). Resolução no 581/2018. Atualiza, no âmbito do Sistema Cofen/Conselhos Regionais de Enfermagem, os procedimentos para Registro de Títulos de Pós - Graduação Lato e Stricto Sensu concedido a Enfermeiros e aprova 
a lista das especialidades [Internet]. Brasília, DF; 2018 [cited 2019 May 07]. Available from: http://www.cofen.gov.br/resolucao-cofenno-581-2018_64383.html

17. Conselho Federal de Enfermagem (COFEN). Resolução no 585/2018. Estabelece e reconhece Acupuntura como especialidade e/ou qualificação do profissional de Enfermagem [Internet]. Brasília, DF; 2018 [cited 2019 May 07]. Available from: http://www.cofen.gov.br/ resolucao-cofen-no-585-2018_64784.html

18. Mendes KDS, Silveira RCCP, Galvão CM. Revisão integrativa: método de pesquisa para a incorporação de evidências na saúde e na enfermagem. Texto Contexto Enferm. 2008;17(4):758-64. doi: 10.1590/S0104-07072008000400018

19. Santos CMC, Pimenta CAM, Nobre MRC. The PICO strategy for the research question construction and evidence search. Rev Latino-Am Enfermagem. 2007;15(3):508-11. doi: 10.1590/S0104-11692007000300023

20. Sociedade Brasileira para Estudo da Dor. Porque a dor é uma questão também de saúde pública [Internet]. 2019 [cited 2019 May 6]. Available from: http://sbed.org.br/duvidas-frequentes-2/dor-no-brasil/

21. Melnyk BM, Fineout-Overholt E. Evidence-based practice in nursing \& healthcare: a guide to best practice. Philadelphia: Wolters Kluwer, Lippincott Williams \& Wilkins; 2011.624 p.

22. Araújo APS, Zampar R, Pinto SME. Auriculoterapia no tratamento de indíviduos acometidos por distúrbios osteomusculares relacionados ao trabalho (dort)/ lesões por esforços repetitivos (ler). Arq Ciên Saúde Unipar. 2006;10(1):35-42. doi: 10.25110/arqsaude.v10i1.2006.140

23. Zanelatto AP. Avaliação da acupressão auricular na Síndrome do Ombro Doloroso: estudo de caso. Rev Bras Enferm. 2013;66(5):694-701. doi: 10.1590/S0034-71672013000500009

24. Hunter RH, McDonough SM, Bradbury I, Liddle SD, Walsh DM, Dhamija S, et al. Exercise and Auricular Acupuncture for Chronic Low-back Pain: a Feasibility Randomized-controlled Trial. Clin J Pain. 2012;28(3):259-67. doi: 10.1097/AJP.0b013e3182274018

25. Martin BR. Multimodal Care in the management of a patient with chronic tendinopathy of the bíceps femoris: a case report. J Chiropr Med. 2017;16(2):156-62. doi: 10.1016/j.jcm.2017.01.006

26. Sator-Katzenschlager SM, Szeles JC, Scharbert G, Michalek-Sauberer A, Kober A, Heinze G, et al. Electrical stimulation of auricular acupuncture points is more effective than conventional manual auricular acupuncture in chronic cervical pain: a pilot study. Anesth Analog [Internet]. 2003 [cited 2019 May 6];97(5):1469-73. Available from: https://mail.vagusdent.gr/images/PDF/Klinikes-dokimes/anesth_ analg_2003-97.pdf

27. Sator-Katzenschlager SM, Scharbert G, Kozek-Langenecker SA, Szeles JC, Finster G, Schiesser AW, et al. The short-and long-term benefit in chronic low back pain through adjuvant electrical versus manual auricular acupuncture. Anesth Analog [Internet]. 2004 [cited 2019 May 6];98(5):1359-64. Available from: https://www.dyansys.com/sites/default/files/anesthesia-acupuncture-back-pain.pdf

28. Ushinohama A, Cunha BP, Costa LOP, Barela AMF, Freitas PB. Effect of a single session of ear acupuncture on pain intensity and postural control in individuals with chronic low back pain: a randomized controlled trial. Braz J Phys Ther. 2016;20(4):328-35. doi: 10.1590/ bjpt-rbf.2014.0158

29. Zhao HJ, Tan JY, Wang T, Jin L. Auricular therapy for chronic pain management in adults: a synthesis of evidence. Complement Ther Clin Pract. 2015;21(2):68-78. doi: 10.1016/j.ctcp.2015.03.006

30. Silva ACO, Biasotto-Gonzalez DA, Dos Santos DM, Melo NC, Gomes CAFP, Amorim CF et al. Evaluation of the immediate effect of auricular acupuncture on pain and electromyographic activity of the upper trapezius muscle in patients with nonspecific neck pain: A randomized, single-blinded, sham-controlled, crossover study. Evid Based Complement Alternat Med. 2015.1-8. doi: 10.1155/2015/523851

31. Suen LKP, Wong EMC. Longitudinal changes in the disability level of the elders with low back pain after auriculotherapy. Complement Ther Med. 2008;16(1):28-35. doi: 10.1016/j.ctim.2007.09.002

32. Suen LKP, Wong TKS, Chung JWY, Yip VYB. Auriculotherapy on low back pain in the elderly. Complement Ther Clin Pract. 2007;13(1):63-9. doi: 10.1016/j.ctcp.2006.10.005

33. Moura CC, Chaves ECL, Chianca TCM, Ruginsk SG, Nogueira DA, lunes DH. Effects of auricular acunpucture on chonic pain in people with back musculoskeletal disorders: a randomized clinical trial. Rev Esc Enferm USP. 2019;53:e03418. doi: 10.1590/s1980-220×2018009003418

34. Garner BK, Hopkinson SG, Ketz AK, Landis CA, Trego LL. Auricular acupuncture for chronic pain and insomnia: a randomized clinical trial. Med Acupunct. 2018;30(5):262-72. doi: 10.1089/acu.2018.1294.

35. Peng D, Yang T, Chen Y, Dong Y, Zhang Y, Shi G, et al. Clinical observation on he's three removing obstruction therapies of acupuncturemoxibustion for 53 cases of lumbar intervertebral disc herniation. International Journal Of Clinical Acupuncture. 2016;25(3):112-6. doi: 10.3103/S1047197916020095.

36. Almeida DC, Kraychete DC. Low back pain- a diagnostic approach. Rev Dor. 2017;18(2):173-7. doi: 10.5935/1806-0013.20170034.

37. Moura CC, lunes DH, Agostinho AAM, Santos N, Silva AM, Chaves ECL. Assessment and correlation between subjective and physiological variables of chronic spinal pain. Rev Dor. 2017;18(3):194-8. doi: 10.5935/1806-0013.20170102.

38. Kurebayashi LFS, Turrini RNT, Souza TPB, Marques CF, Rodrigues RTF, Charlesworth K. Auriculotherapy to reduce anxiety and pain in nursing professionais: a randomized clinical trial. Rev Latino-Am Enfermagem. 2017;25:e2843. doi: 10.1590/1518-8345.1761.2843

39. Moura CC, Carvalho CC, Silva AM, lunes DH, Carvalho EC, Chaves ECL. Auriculoteraia efeito sobre a ansiedade. Rev Cuba Enferm [Internet]. 2015 [cited 2019 May 6];30(2):120-34. Available from: http://scielo.sld.cu/pdf/enf/v30n2/enf05214.pdf 